\title{
A 94 GHz EPR Spectrometer with Fabry-Perot Resonator
}

\author{
E. Haindl and K. Möbius \\ Institut für Molekülphysik, Freie Universität Berlin
}

H. Oloff

Schering AG Berlin

Z. Naturforsch. 40 a, 169-172 (1985); received November 26, 1984

An EPR spectrometer is described working in the $94 \mathrm{GHz}$ (W-band) frequency range. It comprises a Fabry-Perot type microwave resonator connected to a conventional microwave bridge, a phase lock loop for microwave frequency stabilization, and a superconducting magnet. The improved separation of W-band spectra from two sites with only slightly different $g$-factors is demonstrated using an $\alpha$-bromo radical in an X-irradiated 5-bromodeoxyuridine single crystal as example.

\section{Introduction}

There are several situations, where the application of higher microwave frequencies in EPR spectroscopy is favourable in comparison with the commonly used X-band $(9.5 \mathrm{GHz})$ or Q-band $(35 \mathrm{GHz})$ frequencies. Millimeter waves were, for example, used in very early studies of systems with large zero field splittings. These spectrometers either involve the use of backward wave oscillators [1] or harmonic generator crystals [2] for the production of millimeter waves. Other groups are studying organic radicals at frequencies of $70 \mathrm{GHz}$ [3] and $150 \mathrm{GHz}$ [4]. In both cases, the microwave frequency is produced by a klystron, and a cylindrical cavity is used. Even ENDOR has been applied [5] at $70 \mathrm{GHz}$ microwave frequency for the study of radicals produced by ionizing radiation.

In this work a frequency of $94 \mathrm{GHz}$ (W-band), corresponding to a wavelength of $3.2 \mathrm{~mm}$, is used to take advantage of the separation of different sites with different $g$-factors of a radical produced by Xirradiation of a single crystal of 5-bromodeoxyuridine.

In the design of the spectrometer, several requirements were considered: Firstly, it should be possible to investigate samples whose spectra extend over a large range of the magnetic field. Secondly, high

Reprint requests to Dr. E. Haindl, Institut für Molekülphysik, Freie Universität Berlin, Arnimallee 14, D-1000 Berlin 33, West-Germany. microwave field strength at the sample was desirable for a later extension to ENDOR experiments. In addition, easy access to the sample would facilitate future optical experiments.

The resonator used is of the Fabry-Perot type. The dimensions of such a resonator are large in comparison with a cylindrical cavity, it is easily fabricated and it is open from the sides for easy access. Fabry-Perot type resonators have been used in the centimeter and millimeter wave regions for studying dielectric materials and for microwave absorption spectroscopy. Various geometries have been published with mirror distances and diameters ranging from several centimeters to several meters. Design criteria and applications of Fabry-Perot resonators have recently been reviewed [6]. However, there are only very few applications of FabryPerot resonators in magnetic resonance $[7,8]$.

\section{Instrumentation}

A Varian VRB-2111A reflex klystron with maximum output power of $300 \mathrm{~mW}$ is used as the $94 \mathrm{GHz}$ microwave source with Micro-Now model 756 power supply and Micro-Now model 211 frequency stabilizer. A Hewlett-Packard $8672 \mathrm{~A}$ frequency synthesizer is used as reference frequency. All components of the W-band bridge are from Hughes (isolator $44606 \mathrm{H}, 20 \mathrm{~dB}$ directional coupler $45326 \mathrm{H}$, harmonic mixer $47436 \mathrm{H}$, attenuator $45726 \mathrm{H}$, circulator $45166 \mathrm{H}$ and broadband detec- 
tor $47316 \mathrm{H}$ ). A superconducting magnet (Oxford Instruments 250/89) produces the variable magnetic field up to $5.87 \mathrm{~T}$. Details of the magnetic field regulation are described elsewhere [9].

\section{Fabry-Perot Resonator}

The mechanical set-up of the Fabry-Perot resonator is shown in Figure la. Two plane-concave spherical mirrors are supported in a cylindrical resonator body. The mirror distance can be tuned by a fine thread to adjust the resonator frequency to the klystron frequency. The resonance frequency variation is $10 \mathrm{MHz}$ for a mirror distance change of $1 \mu \mathrm{m}$. Large windows in the resonator body allow for easy access from all sides.

The longitudinal distribution of the $\mathrm{B}_{1}$ field along the resonator axis and its radial distribution is shown in Figure $1 \mathrm{~b}, \mathrm{c}$. The distribution of the millimeter wave field inside the resonator is calculated according to the formulas derived for laser resonators [10]. Parameters are the radii $R_{1}=R_{2}=R$ of the two concave mirrors, their distance $d$, the mirror diameter $2 a$ and the wavelength $\lambda$ of the radiation field. Stability for microwave resonance is obtained for

$$
0<\left(1-d / R_{1}\right)\left(1-d / R_{2}\right)<1 .
$$

The fundamental $\mathrm{TEM}_{00 q}$ mode has a Gaussian radial field distribution. The beam radius $w$ at the

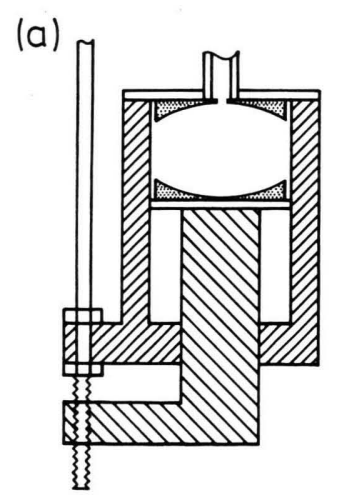

(b)

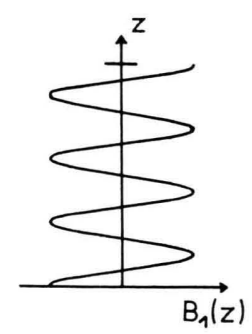

(c)

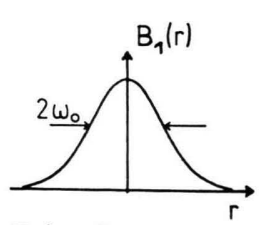

Fig. 1. Mechanical set-up (a) of the Fabry-Perot resonator with resonator mirrors, waveguide connection, and frequency tuning mechanism; longitudinal (b) and radial (c) distribution of the microwave $\mathrm{B}_{1}$ field (first order calculation). mirrors, which is defined as the distance where the field is reduced to a value of $1 / e$ of the maximum value at the axis, is given by

$$
w^{2}=(\lambda R / \pi) /(2 R / d-1)^{1 / 2} .
$$

The beam radius $w_{0}$ in the plane in the center between the two mirrors is calculated from the equation

$$
w_{0}^{2}=(\lambda / 2 \pi)[d(2 R-d)]^{1 / 2} .
$$

Diffraction losses are determined by the mirror diameter.

The values chosen for the mirror radii are $R=16 \mathrm{~mm}$, their distance is $d \approx 10 \mathrm{~mm}$ and the mirror diameter is $2 a=18 \mathrm{~mm}$. The calculated beam radii are $w=3.6 \mathrm{~mm}$ at the mirrors and $w_{0}=2.8 \mathrm{~mm}$ in the plane in the middle between the mirrors. The mirror distance corresponds to a $\mathrm{TEM}_{00 q}$ mode with $q=6$. The sample centering in a minimum of the electric field in the middle of the resonator is adjusted by moving the resonator and keeping the sample holder fixed. The $Q$-factor of the resonator is $Q \approx 10000$. The $\mathrm{TEM}_{00 q}$ mode is linearly polarized, the polarization plane is determined by the polarization of the microwave field inside the waveguide.

Two different types of resonator mirrors have been used. In the first experiments, the mirrors consisted of suprasil quartz with a coating of $1 \mu \mathrm{m}$ of silver and $0.1 \mu \mathrm{m}$ of gold. The resonator was coupled to the microwave field by a hole in the center of the top mirror. The coupling efficiency could be adjusted by varying the distance from the waveguide to the resonator mirror. The second version now used in our experiments consists of brass mirrors with a coupling hole about $0.8 \mathrm{~mm}$ in diameter and $10 \mu \mathrm{m}$ thick. This version is more easily coupled to the waveguide, coarse tuning of the coupling is done in the usual way by adjusting the hole diameter. Fine tuning is accomplished by use of a slide screw tuner close to the resonator coupling hole.

\section{Microwave Bridge and Frequency Control}

A schematic diagram of the microwave bridge and the klystron frequency control is given in Figure 2. The basic design of the W-band microwave bridge is the same as for a standard X-band 


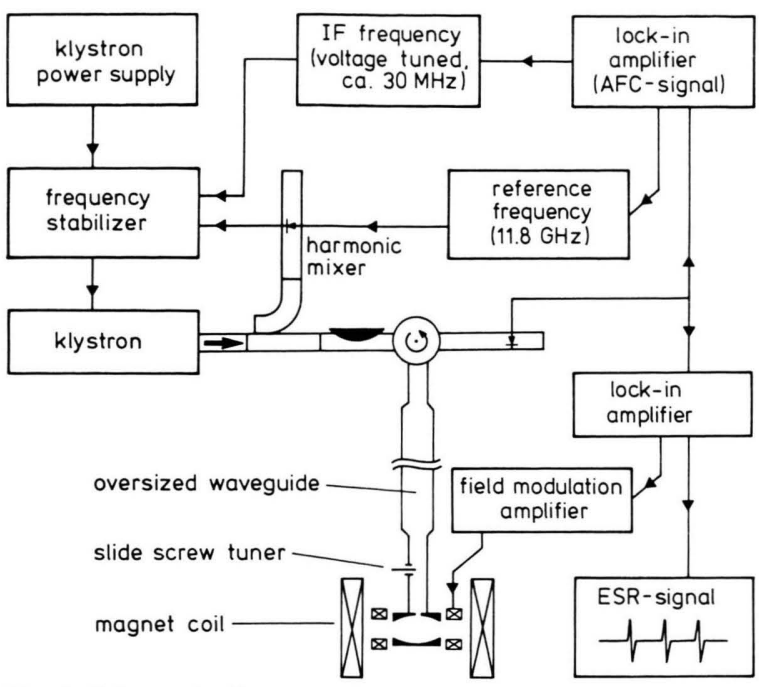

Fig. 2. Schematic diagram of microwave bridge and microwave frequency control circuit.
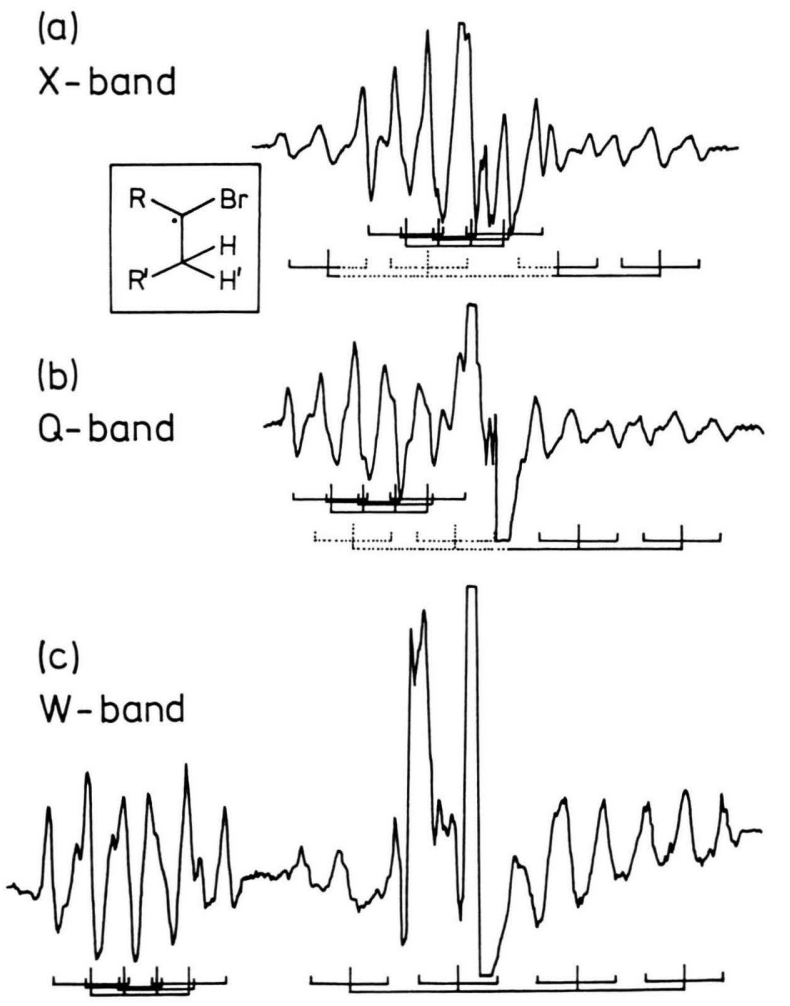

Fig. 3. Spectra of an $\alpha$-bromo radical at X-band (a), Q-band (b) and W-band (c) frequencies. The splitting pattern of the two sites is due to hyperfine interaction of an $\alpha$-bromo nucleus and two protons in $\beta$-position and is indicated by stick diagrams [11]. bridge. An oversized WR-28 waveguide is used between circulator and resonator to reduce microwave power losses by an order of magnitude.

It is estimated that the power at the resonator input is only about $10 \%$ of the klystron output power due to high losses of all microwave components at this high frequency. Resonator and EPR sample are placed inside a cryostat for sample temperature variation. A bucking arm was not installed because of phase stability problems.

Great care has been taken to ensure good klystron frequency stability which is important in the case of narrow line spectra. The klystron is phase locked to the $8^{\text {th }}$ harmonic of a microwave synthesizer. The frequency stabilizer compares the $30 \mathrm{MHz}$ IF output of the harmonic mixer to an external IF frequency reference to correct the klystron reflector voltage. This phase lock loop (PLL) circuit provides exact klystron frequency determination and good spectral purity, the bandwidth of the mixer IF output is less than $1 \mathrm{kHz}$. In addition, an AFC circuit is installed to tune the klystron to the resonator frequency. This AFC tuning is achieved by variation of the two reference frequencies used in the PLL circuit. Coarse tuning is done by stepping the microwave synthesizer with a $2 \mathrm{kHz}$ resolution, giving $16 \mathrm{kHz}$ steps for the klystron frequency. A voltage controlled oscillator (VCO) is used as IF reference frequency for fine tuning by small variations around $30 \mathrm{MHz}$. The AFC signal is derived from a lock-in amplifier, with klystron frequency modulation achieved by modulating the $30 \mathrm{MHz}$ IF reference.

\section{Application}

High frequency EPR spectroscopy can be useful in various situations, for instance for large zero field splittings, for small single crystal samples, or for the separation of overlapping spectra of organic radicals with only slightly different $g$-factors.

An example of the last type of situations is shown in Figure 3. The overlapping spectra are essentially due to an $\alpha$-bromo radical, which is produced by $\mathrm{X}$-irradiation of a single crystal of 5-bromodeoxuridine. The crystal contains two magnetically inequivalent sites and, as a consequence, the assignment of lines, the evaluation of the spectral parameters, and the identification of the radical structure were only 
possible by comparing the spectra at X-band and at Q-band frequencies [11]. These spectra are shown in Figure $3 \mathrm{a}$ and in Figure $3 \mathrm{~b}$. The $g$-factor anisotropy is not sufficiently large to separate the spectra of the two crystal sites at these frequencies.

The situation is much better at W-band frequencies. Figure $3 \mathrm{c}$ shows the W-band spectrum, taken for the same crystal orientation. The separation of the spectra is sufficient to allow for an easy determination of the spectral parameters of the two sites.

The spectrometer has been tested with different liquid and solid samples. Typical linear crystal

[1] J. B. Mock, Rev. Sci. Instrum. 31, 551 (1960).

[2] P. R. Elliston, G. J. Troup, and D. R. Hutton, J. Sci. Instrum. 40, 586 (1963).

[3] H. C. Box, Radiation Effects, ESR and ENDOR Analysis, Academic Press, New York 1977, pp. 101.

[4] M. A. Ondar, A. A. Dubinskii, O. Ya. Grinberg, J. A. Grigor'ev, L. B. Volodarskii, and Ya. S. Lebedev, Zhurnal Strukturnoi Khimii 22, 59 (1981).

[5] M. J. Colaneri, E. E. Budzinski, and H. C. Box, J. Magn. Res. 44, 580 (1981).

[6] G. W. Chantry, J. Phys. E15, 3 (1982); R. N. Clarke and C. B. Rosenberg, J. Phys. E15, 9 (1982); E. A. M. Baker and B. Walker, J. Phys. E15, 25 (1982). sample dimensions are $0.5 \mathrm{~mm}$, capillary tubes of $0.3 \mathrm{~mm}$ diameter are used for liquids. The sensitivity, determined with a $10^{-4} \mathrm{~m}$ solution of tanol in toluene is $1 \times 10^{9}$ spins/Gauss. The magnetic field regulation long term stability is $10 \mathrm{mG} / \mathrm{min}$, the short term stability at a time constant of $1 \mathrm{sec}$ is $1 \mathrm{mG}$, the smallest detected linewidth was $50 \mathrm{mG}$ [9].

\section{Acknowledgement}

This work was supported by the Deutsche Forschungsgemeinschaft (Sfb 161).

[7] I. Amity, Rev. Sci. Instrum. 41, 1492 (1970).

[8] H. W. van Kesteren, W. Th. Wenckebach, J. Schmidt, and N. J. Poulis, Proceedings of the XXII ${ }^{\text {nd }}$ Congress Ampère 1984, pp. 275.

[9] O. Burghaus, E. Haindl, M. Plato, and K. Möbius, submitted to J. Phys. E.

[10] H. Kogelnik and T. Li, Appl. Optics 5, 1550 (1966).

[11] E. Haindl and J. Hüttermann, J. Magn. Resonance 30, 13 (1978). 